\title{
Non-Covalent Hydrogels of Cyclodextrins and Poloxamines for the
}

\section{Controlled Release of Proteins}

\author{
Eneko Larrañeta, José Ramón Isasi* \\ Departamento de Química y Edafología, Facultad de Ciencias, \\ Universidad de Navarra, 31080 Pamplona, Navarra, Spain
}

doi:10.1016/j.carbpol.2013.11.002

(C) 2013. This manuscript version is made available under the CC-BY-NC-ND 4.0 license http://creativecommons.org/licenses/by-nc-nd/4.0/

* Corresponding author. Tel.: +34-948-425600; fax: +34-948-425740.

E-mail address: jrisasi@unav.es (J.R. Isasi). 
1 ABSTRACT: Different types of gels were prepared by combining poloxamines (Tetronic), i.e.

2 poly(ethylene oxide)/poly(propylene oxide) (PEO/PPO) octablock star copolymers, and

3 cyclodextrins (CD). Two different poloxamines with the same molecular weight (ca. 7000) but

4 different molecular architectures were used. For each of their four diblock arms, direct Tetronic

5904 presents PEO outer blocks while in reverse Tetronic 90R4 the hydrophilic PEO blocks are

6 the inner ones. These gels were prepared by combining $\alpha-C D$ and poloxamine aqueous solutions.

7 The physicochemical properties of these systems depend on several factors such as the structure

8 of the block copolymers and the Tetronic/ $\alpha-\mathrm{CD}$ ratio. These gels were characterized using

9 differential scanning calorimetry (DSC), viscometry and X-ray diffraction measurements. The

10 90R4 gels present a consistency that makes them suitable for sustained drug delivery. The

11 resulting gels were easily eroded: these complexes were dismantled when placed in a large

12 amount of water, so controlled release of entrapped large molecules such as proteins (Bovine

13 Serum Albumin, BSA) is feasible and can be tuned by varying the copolymer/CD ratio.

\section{Highlights}

16 - Direct and reverse poloxamines yield supramolecular gels when interacting with $\alpha$ 17 cyclodextrin in aqueous media, but solid precipitates are obtained in the case of $\beta$ $18 \quad$ cyclodextrin.

19 - The rheological behaviour of the poloxamine/ $\alpha$-cyclodextrin gels depend on the 20 copolymer/ oligosaccharide ratio and the temperature.

21 - Proteins or other molecules can be loaded into these supramolecular structures before 22 gelation.

23 - The resulting gels were easily eroded so controlled release of entrapped proteins is 24 feasible and can be tuned by varying the copolymer/CD ratio. 


\section{Keywords}

27 Cyclodextrins, self-assembly, host-guest interactions, controlled release

\section{INTRODUCTION}

30 Hydrogels are crosslinked polymeric networks with a soft and hydrophilic nature that makes

31 them suitable in numerous biomedical applications such as matrices for drug delivery, wound

32 healing, or tissue engineering (Ko et al., 2013). The self assembled hydrogels, i.e. those

33 possessing a physical crosslinking, have attracted much attention lately. These hydrogels can be

34 useful in pharmaceutical and biological applications, especially for some purposes as gene

35 carriers or in the delivery of delicate bioactive agents such as drugs or proteins (Koopmans and

36 Ritter, 2008, Li et al., 2006, Li and Loh, 2008, Lin et al., 2013, Ni et al., 2009, Nielsen et al.,

37 2009, van de Manakker et al., 2008, Van Tomme et al., 2008, Wintgens et al., 2008). Among

38 their promising properties, they present a quick degradation process and the capability of forming

39 the gel "in situ" by physical crosslinking between molecules coming from different solutions. In

40 this process of gelation, a protein, a peptide, or a drug can be added to the mixture so it will

41 finally become immobilized inside the bulk of the gel (Gref et al., 2006, Li et al., 2006). This

42 particular property is very significant because it allows us to inject the mixture inside a tissue

43 using a simple needle without any kind of surgery (Li et al., 2003a).

Amphiphilic molecules such as polyethylene oxide/polypropylene oxide (PEO/PPO)

45 block copolymers exhibit interesting self-assembling properties. The behaviour of amphiphilic

46 triblock PEO-PPO-PEO copolymers (poloxamers or Pluronics) can be tailored by modifying the

47 molecular weight and the PEO/PPO ratio within the copolymer (Alexandridis and Hatton, 1995,

48 Trong et al., 2008). If the distribution of the hydrophilic and hydrophobic blocks is inverted, the 
resulting PPO-PEO-PPO poloxamers (Pluronic R or reverse) show a different behaviour in water

50 solutions (Zhou and Chu, 1994).

51 Tetronics or poloxamines are four armed copolymers that contain two blocks per arm, a

52 PEO block and a PPO block. Normal or direct Tetronics present outer PEO blocks, while reverse

53 Tetronics posses inner PEO blocks. The behaviour of normal Tetronics in water has already been

54 reported (Na et al., 1999) although only a couple of works deal with reverse Tetronics (Plestil et

55 al., 2003, Larrañeta and Isasi, 2013). Some Tetronics can form aggregates such as micelles and,

56 in some instances, they can gel under certain conditions, so poloxamines display a great potential

57 as components of drug delivery systems and tissue engineering devices (Alvarez-Lorenzo et al.,

58 2007, Gonzalez-Lopez et al., 2008).

In previous works, some authors have discovered that mixing PEO/PPO block copolymers

60 with cyclodextrins (CD) produces self-assembled gels (Koopmans and Ritter, 2008, Li et al.,

61 2006, Nielsen et al., 2009, Tan et al., 2012). Cyclodextrins are natural molecules derived from

62 starch with a relatively hydrophobic cavity, which can form inclusion complexes with different

63 molecules, including linear polymers (Dodziuk, 2006). In this case, the resulting complex is

64 called a polypseudorotaxane (Li et al., 2003a, Li et al., 2006, Ni et al., 2009). For instance, $\alpha$-CD

65 molecules yield inclusion complexes with PEO and its block copolymers (Harada et al., 1995,

66 Harada, 1998, Harada et al., 2009, Harada, 1997, Li et al., 2001). On the other hand,

67 cyclodextrins with a wider rim (i.e. $\beta$ - and $\gamma$-CD) are capable of forming complexes with PPO 68 blocks.

69 Several works regarding the formation of inclusion complexes and gels between 70 poloxamers (Pluronics) and CDs have been published (Li et al., 2003b, Li and Loh, 2008, Ni et

71 al., 2009). The complexes between normal poloxamines (Tetronics) and CDs have been recently

72 studied (Simões et al. 2013). In this work, we have used two octablock PEO/PPO copolymers:

73 Tetronic 904 and its corresponding reverse version, Tetronic 90R4. These two medium sized 
74 copolymers have practically the same block lengths (16-18 units), but a different architecture.

75 Our main purpose is to ascertain whether the properties of the obtained gels depend on these

76 architectures. After their physical characterization, the gels were tested as controlled release

77 matrices by evaluating their erosion in water media and the release kinetics of a model protein,

78 Bovine Serum Albumin (BSA).

80 EXPERIMENTAL SECTION

81 Materials. Tetronic 90R4 is a viscous yellow liquid, whose molecular weight given by the

82 manufacturer is $\mathrm{M}_{90 \mathrm{R} 4}=6900 \mathrm{~g} / \mathrm{mol}$. Its chemical composition was determined by ${ }^{1} \mathrm{H}-\mathrm{NMR}$

83 (Bruker DPX 300): $\mathrm{PO}_{16} \mathrm{EO}_{18}$ per arm (see Figure A1, Supplementary data). Tetronic 904 is a

84 colorless viscous paste with a molecular weight of $\mathrm{M}_{90 \mathrm{R} 4}=6700 \mathrm{~g} / \mathrm{mol}$. The composition of the

85 blocks was also determined by ${ }^{1} \mathrm{H}-\mathrm{NMR}$ : $\mathrm{PO}_{15} \mathrm{EO}_{19}$ per arm. According to their safety data sheets

86 (BASF), these products are non-irritant to the skin, practically nontoxic for dermal applications,

87 and show a low oral toxicity (LD50 $>5,000 \mathrm{mg} / \mathrm{kg}$ ). Both cyclodextrins, $\alpha-\mathrm{CD}$ and $\beta-\mathrm{CD}$, were

88 obtained from Wacker Chemie AG and were used without further purifications. Bovine Serum

89 Albumin (BSA) was purchased from Sigma-Aldrich and used as received.

90 Preparation of complexes and gels. All complexes were formed mixing a determinate amount

91 of Tetronic with an aqueous solution of $\mathrm{CD}$ followed by vigorous stirring. In the case of

92 poloxamine 904, the copolymer paste was dissolved in a certain amount of water prior to its

93 mixing with the $\mathrm{CD}$ solutions, in order to facilitate the homogenization process. Before any

94 measurements, the resulting mixtures were kept at room temperature for at least one night. In the

95 case of the $\alpha$-CD mixtures, the viscous fluids were used as obtained, without further purifications.

96 On the other hand, for $\beta$-CD complexes, a white powder appears at the bottom of the flask when

97 the two solutions are mixed. The supernatant was carefully removed and the powder precipitate 
was freeze-dried and washed with tetrahydrofuran (THF) in order to remove the excess of

99 uncomplexed poloxamine (Harada, 1998).

100 Phase diagrams. A series of Tetronic/ $\alpha-C D /$ water gel samples were prepared in $8 \mathrm{~mL}$ vials

101 varying the $\alpha-\mathrm{CD} /$ copolymer ratio. The samples were placed in a water bath equipped with a

102 thermostatic head. The state of the samples was evaluated for each temperature by direct

103 observation. It was characterized as "gel" or "sol" according to its fluidness when the flask was

104 kept inverted during at least $10 \mathrm{~s}$. Cylindrical $23 \mathrm{~mm}$ diameter glass vials containing 5 g samples

105 were used for this analysis. The influence of experimental conditions on the results of this

106 experimental procedure was discussed in a previous work (Larrañeta and Isasi, 2012). The

107 temperature ranged between $10^{\circ} \mathrm{C}$ and $60{ }^{\circ} \mathrm{C}\left( \pm 0.1{ }^{\circ} \mathrm{C}\right)$, using $5{ }^{\circ} \mathrm{C}$ steps; a stabilization time of

10810 minutes was considered as an appropriate delay time prior to testing the mixtures at each

109 temperature.

110 Wide-angle X-ray diffraction. The diffractograms of $\beta-\mathrm{CD}$ complexes and $\alpha-\mathrm{CD}$ gels were

111 carried out in a Brüker D8 Advance X ray diffractometer equipped with a X ray generator,

112 Kristalloflex $\mathrm{K} 760$, using the radiation $\mathrm{K}_{\alpha 1}$ of the $\mathrm{Cu}(\lambda=1.5417 \AA)$, and a scanning speed of $0.4^{\circ}$

113 per minute. The $\alpha-\mathrm{CD} /$ Tetronic hydrogels were vacuum dried at $60^{\circ} \mathrm{C}$ overnight prior to the

114 measurements.

115 Viscosity measurements. The viscosity of the $\alpha-C D /$ copolymer hydrogels was evaluated using a

116 Haake Viscotester 550 rotational viscometer equipped with a thermostatic bath Thermo Phoenix

117 II. Two rotors were used: the SV rotor was used for all 90R4/CD mixtures and for those 904/CD

118 mixtures with higher viscosities, and the NV was used for 904/CD mixtures with a low viscosity

119 (gels with $10 \%$ and $15 \%$ of the poloxamine). All measurements were performed at $25^{\circ} \mathrm{C}$ and the

120 range of shear rates covered was between 5 and $40 \mathrm{~s}^{-1}$. After a stabilization time of 30 seconds, an

121 average of 100 data points was recorded during a measuring period of 60 seconds. 
122 DSC analyses. The thermal analysis of the gels was performed using a DSC Mettler TA4000.

123 The samples were kept at $140^{\circ} \mathrm{C}$ for 20 min to remove the excess water. Two scans were

124 registered between $-100^{\circ} \mathrm{C}$ and $140{ }^{\circ} \mathrm{C}$ using a scan speed of $20^{\circ} \mathrm{C} / \mathrm{min}$. Glass transition

125 temperatures were calculated as the half-height of the corresponding heat-capacity jump. All

126 reported values were determined in the second DSC run.

127 In vitro release and erosion kinetics. The release studies were performed from $500 \mathrm{~mL}$ of $\mathrm{pH} 7$

128 phosphate buffer solutions using a SOTAX AT 7 Smart USP dissolution testing device at $37^{\circ} \mathrm{C}$

129 and $25 \mathrm{rpm}$ stirring speed. The gels $(20 \mathrm{~g})$ were formed at the bottom of the device vessels by

130 mixing $14.3 \mathrm{~g}$ of a $14 \% \alpha-\mathrm{CD}$ solution (including $150 \mathrm{mg}$ of BSA) and the appropriate amounts

131 of poloxamine and water needed to reach the required gel compositions. Then, the dissolution

132 medium was carefully added to the top at the beginning of the release kinetics. Aliquots $(5 \mathrm{~mL})$

133 were withdrawn according to a sampling time program of about $10 \mathrm{~h}$, and these sample volumes

134 were replaced with fresh medium. The BSA concentration in each sample was evaluated both by

135 UV-vis (Hewlett Packard 8452A) and fluorescence spectrometries (Perkin Elmer LS 50 B). Both

136 the poloxamine and the substrate (BSA) absorb in the same UV region; in addition, the

137 fluorescence of BSA is quenched by the poloxamine that is also released as the matrices are

138 eroded. Therefore, a combination of the results obtained from these two techniques had to be

139 used in order to determine the amount of substrate released as a function of time (see

140 Supplementary data). $\alpha-C D$, which is also present in the solution during the erosion process, is

141 not a quencher of BSA. The amount of $\alpha-C D$ released from the matrix was evaluated using size

142 exclusion chromathography (SEC) (Waters 600E system equipped with a Waters 2414 Refractive

143 Index detector and an Aquagel $\mathrm{OH}-30$ column). The erosion kinetic curves were constructed

144 evaluating the amount of $\alpha-C D$ dissolved in the samples as a function of time. Acidic and basic

145 media were prepared using $\mathrm{HCl} 0.1 \mathrm{M}$ or $\mathrm{NaOH} 0.1 \mathrm{M}$, respectively. 
148 Complexation between cyclodextrins and 90R4 or 904. When 90R4 or 904 poloxamine

149 aqueous solutions are mixed with $\alpha$-CD solutions in certain ratios, a viscous white hydrogel is

150 formed. The evidence of complexation between EO blocks (either in PEO homopolymer or in its

151 copolymers) and $\alpha$-CD molecules is a well known fact (Larrañeta and Isasi, 2012, Li et al.,

152 2003b, Ni et al., 2009). Both the complexed EO units and the uncomplexed PO blocks tend to

153 self-associate, the first ones by interactions between the CD moieties and the second ones by

154 hydrophobic interactions (see Figure 1). Hydrophobic associations seem to be more difficult in

155 the case of Tetronic 904 once PEO are threaded due to steric hindrance. On the other hand,

156 associations between PPO blocks threaded by $\alpha-C D$ would be possible in both instances. By

157 varying the $\alpha-\mathrm{CD} /$ copolymer ratio and the amount of water, two different types of aggregates can

158 be obtained: either white viscous fluids or gels that remain stable when the flask is inverted. In

159 addition, it has to be pointed out that the formation process is considerably faster for the normal

160 poloxamine (904) than for the reverse one (90R4), a result attributed to the disposition of the

161 PEO blocks in the copolymers. For the reverse poloxamine, the PEO blocks are located in the

162 inner part of the chains so $\alpha$-CD molecules must surpass the PPO blocks in order to reach the

163 PEO blocks and form the resulting polyrotaxane type structures, as occurs for PPO-PEP-PPO

164 copolymers ( $\mathrm{Li}$ et al., 2003b). In the case of 904 star octablock copolymers, the PEO blocks are

165 in the outer part of the chains so the kinetics of complexation are faster. For 904 the process

166 takes place in a few seconds, while in the case of 90R4 complexes, they are formed within 20

167 minutes.

168 Figures 2 and 3 show the sol-gel phase diagrams for the systems 90R4/ $\alpha-C D$ and 904/ $\alpha-$

$169 \mathrm{CD}$, respectively (the solubility limit for $\alpha-\mathrm{CD}$ aqueous solution is ca. $14 \mathrm{wt} \%$ ). Tetronic $90 \mathrm{R} 4$

170 can not gel by itself in water solutions (Larrañeta and Isasi, 2013, Plestil et al., 2003) in contrast

171 to Tetronic 904, which forms gels under certain conditions of concentration and temperature 
172 (Alvarez-Lorenzo et al., 2007) (above 40\% wt., see Figure A2, Supplementary data). As can be

173 seen in the phase diagrams, at least $5 \%(\mathrm{w} / \mathrm{w})$ of $\alpha-\mathrm{CD}$ is needed to produce gels for both normal

174 and reverse poloxamines. Interestingly, this threshold value matches the one found for a

175 considerably larger normal poloxamine, Tetronic 908, with $\mathrm{PO}_{21} \mathrm{EO}_{114}$ per arm (Simões et al.,

176 2013). It is noticeable that, for intermediate $\alpha-C D$ concentrations $(5 \%-7 \%)$, the gel zone for the

177 reverse Tetronic mixtures corresponds to higher $90 \mathrm{R} 4 / \alpha-\mathrm{CD}$ ratios. In contrast, for the direct

178 Tetronic (Figure 3), the gel zone appears at the left hand side of the diagrams, i.e. for lower

$179904 / \alpha-C D$ ratios. As can be seen in Figure 1, the outer PEO blocks can be threaded with higher

180 amounts of cyclodextrin with a low steric hindrance. Then, the interaction between the resulting

181 rotaxane structures is easily achieved through the favourable interactions between CD molecules.

182 For the mixture between $10 \%$ of $\alpha-\mathrm{CD}$ with Tetronic 904 , the phase diagram shows a second gel

183 region at the right hand side of the phase diagram. An additional mechanism for gel formation

184 comes into play: the hydrophobic interaction between the excess of uncomplexed PPO blocks. In

185 the case of the reverse poloxamines, $\alpha$-CD threads their inner PEO blocks. A few CD moieties

186 (i.e. lower $904 / \alpha-C D$ ratios, left hand side of the diagrams) suffice to disentangle the octablock

187 chains to yield structures that gellify by hydrophobic interactions between PPO blocks. This is

188 indeed the case for reverse Pluronics (PPO-PEO-PPO triblock copolymers) (Larrañeta and Isasi, 189 2012).

190 An additional consideration regarding the preparation of the samples is needed. Once the 191 mixtures have been heated to analyze their sol-gel behaviour, they were cooled down to room

192 temperature. In some instances, a second heating scan yielded different results, producing gel-like 193 mixtures instead of fluid sols (see Figure A3, Supplementary data). Nevertheless, these 194 thickening effects can be considered quite subtle for 904 gels and they are not too significant for 195 90R4 gels either. 
When aqueous 90R4 or 904 octablock copolymers are mixed with $\beta$-CD solutions, no gels

197 are obtained but solid precipitates. As we have recently shown, the combination of PEO/PPO

198 linear triblock copolymers (i.e. poloxamers or Pluronics) with $\beta$-CD leads also to the formation of

199 a crystalline precipitate powder (Larrañeta and Isasi, 2012).

200 In contrast to the gel formation observed for $\alpha-\mathrm{CD}$ and PEO/PPO copolymers, the mixing

201 of the latter with $\beta$ - or $\gamma$-CD aqueous solutions yields white precipitates with a certain crystalline

202 order (Harada et al., 1995, Li et al., 2001). According to the literature, these precipitates are

203 formed by the inclusion of the PPO blocks inside the $\beta-C D$ cavities producing a

204 polypseudorotaxane type structure (Harada, 1998) yielding self-associated structures such as

205 nanoplatelets (Perry et al., 2011, Tsai et al., 2010).

206

Although colloidal at first, the $\beta-\mathrm{CD} /$ Tetronic aggregates coalesce in a few minutes. The

207 kinetics of the process depends on the poloxamine and it is faster for the 90R4 (see

208 Supplementary data, Figure A4). In the case of $904 / \beta-C D$, the PPO blocks are located in the inner

209 part of the poloxamine arms, so $\beta$-CD needs an additional time to pass through the PEO external

210 blocks. These complexes present a crystalline character that can be evaluated by means of X-ray

211 diffraction (see Supplementary data, Figure A5). Using NMR and elemental analysis, it has been

212 found that they posses a defined stoichiometry of about two propylene oxide units per CD

213 molecule (see Supplementary data, Table A1), an optimal value already reported for other PPO

214 polymers and copolymers (Harada et al., 1995). The characterization study of these complexes

215 can be found in the Supplementary data section.

216 When PEO/PPO block copolymers and CDs are combined, different associated systems

217 can be obtained. Using $\alpha-C D$, two types of associations are present in the mixture: (1)

218 hydrophobic interactions between PPO uncomplexed blocks, and (2) associations between $\alpha$-CD

219 moieties encapsulating PEO complexed blocks. As a result, firm gels are produced (Larrañeta and

220 Isasi, 2012, Ni et al., 2009). In contrast, the hydrophobic interactions are not present in the 
mixtures of these PEO/PPO copolymers with an excess of $\beta$ - or $\gamma$-CD because the PPO blocks are complexed and the hydrophilic PEO blocks do not self-associate in aqueous solutions (Li et al., 223 2006).

Thus, it becomes evident that the type of cyclodextrin determines the gel character of the aggregates. Despite of their unfavourable molecular architecture, the complexes of these star-like copolymers obtained using $\beta-\mathrm{CD}$ are not gelatinous but crystalline precipitates. In contrast, in the case of $\alpha-\mathrm{CD} /$ Tetronic systems, the resulting hydrogels can be suitable for sorption and delivery applications. Obviously, these non-covalent interactions can be easily disrupted when the gels or the complexes are in contact with a large amount of water (Li et al., 2001). This behaviour can be proved to be very useful for some applications such as drug release because the gel matrix will be totally eroded in a short period of time. Prior to testing these gels as protein delivery matrices, their physical properties need to be discussed.

233 Physical properties of poloxamine/a-cyclodextrin gels. The rheological behaviour of 234 poloxamine/ $\alpha-C D$ mixtures has been analyzed using a rotational viscometer. All the samples contain 10\% (w/w) of $\alpha-\mathrm{CD}$ and different concentrations of reverse 90R4 and direct 904 Tetronic. We have chosen this $\alpha$-CD composition as a standard value, between the solubility limit of the cyclodextrin (14\%) and lower compositions which yield mostly solutions instead of gels (7\% and below). These mixtures exhibit a pseudoplastic behaviour, i.e. the viscosity of the samples decreases when the shear rate is increased (see Supplementary data, Figures A6-A7). Figure 4 shows the viscosities measured at a constant shear rate of $40 \mathrm{~s}^{-1}$ as a function of the copolymer concentration for gels containing the same amount of $\alpha-\mathrm{CD}(10 \mathrm{wt} \%)$. Interestingly,

242 both plots show a cusp at about $20 \%(\mathrm{w} / \mathrm{w})$ of the copolymer. This copolymer/CD ratio 243 corresponds, in both cases, to four CDs molecules per poloxamine molecule, i.e. a single $\alpha$-CD 244 molecule per PEO complexing block. This was also the case for a linear PEO/PPO copolymer, 245 Pluronic 10R5, that was previously studied by our group (Larrañeta and Isasi, 2012). Once each 
246 PEO block is, on average, threaded to one cyclodextrin moiety, the conformation of the Tetronic 247 arms change, making the self-assembling process feasible. An excess of uncomplexed 248 poloxamine molecules (higher Tetronic/CD ratios) contributes to an increase in the viscosity of 249 the mixture. Thus, it becomes evident why more "ordered" structures, corresponding to mixtures 250 with less than a $20 \%$ of the poloxamine, yield lower viscosity values.

251 Although they present the same singular point (cusp), the viscosity range of 90R4 gels is 252 significantly higher than that of 904 gels. The different distribution of the blocks in both 253 copolymers can explain this difference. For 90R4, the outer PPO blocks can establish PPO-PPO 254 intermolecular interactions easily, contributing to a remarkable increase in the viscosity of the 255 bulk. On the other hand, the 904 inner PPO blocks can also establish hydrophobic intermolecular 256 interactions, but in a more restrained way.

257 A comparison between XRD patterns for different $904 / \alpha-C D$ and $90 \mathrm{R} 4 / \alpha-\mathrm{CD}$ gels with 258 the polyrotaxane complex formed between a PEO homopolymer $\left(\mathrm{M}_{\mathrm{w}}=400\right)$ and $\alpha-\mathrm{CD}$ is shown 259 in Figure 5. The inclusion complex formed between pure PEO and this cyclodextrin yields a 260 white powder with a channel type structure for $\alpha-\mathrm{CD}$ (Harada et al., 1992). This structure is 261 clearly detected in the XRD pattern with a characteristic peak located at about $20^{\circ}$, and 262 corresponds to the hexagonal packing (210) of the channel structure (Chung et al., 2007). Figure 2635 shows that the same peak is observed for both 904 and 90R4 gels. Although the amorphous 264 halo is, in both cases, considerably large, the diffraction peaks show that there is some degree of 265 order in the gel structure, attributable to the formation of EO/ $\alpha-\mathrm{CD}$ complexes that become 266 aggregated into ordered domains. It has to be pointed out that both gels were prepared using $25 \%$ $267(\mathrm{w} / \mathrm{w})$ of the copolymer and $10 \%(\mathrm{w} / \mathrm{w})$ of $\alpha-\mathrm{CD}$, so there is, on average, less than one CD per 268 PEO block in both cases. Thus, the hexagonal packing of the CD units detected by XRD implies 269 side-to-side packing of single rotaxane structures. 
271 of their complexation with the cyclodextrin moieties (Ni et al., 2009). Table 1 shows the glass

272 transition temperatures for complexes of 904 and $90 \mathrm{R} 4$ with $\alpha-\mathrm{CD}$ for different poloxamine/CD

273 ratios. As can be seen, the complexes formed between 90R4 and $\alpha$-CD do not behave in the same

274 way as $904 / \alpha-C D$ ones. It was previously shown that only mixtures formed between linear

$275 \mathrm{PEO} / \mathrm{PPO}$ copolymers and $\alpha$-CD corresponding EO/CD mole ratios above 2 show a defined $\mathrm{T}_{\mathrm{g}}$

276 (Ni et al., 2009),(Larrañeta and Isasi, 2012). This characteristic value corresponds to the two EO

277 units that fit inside a CD cavity. When the EO/CD ratio is below 2, all the PEO blocks are

278 threaded within CD cavities. These complexed blocks tend to aggregate into ordered domains so

279 the mobility of the chains is very restricted. Table 1 shows that, for EO/CD ratios higher that 2.7,

280 Tetronic 904 complexes show a defined $\mathrm{T}_{\mathrm{g}}$, similar to that of the pure poloxamine. No glass

281 transitions are detected for mixtures below this ratio (i.e. those with an excess of CD moieties). In

282 contrast, Tetronic 90R4 samples show a clear $\mathrm{T}_{\mathrm{g}}$ (with a value close to that of the pure

283 poloxamine) for all the studied $\mathrm{EO} / \mathrm{CD}$ ratios. Because of their molecular architecture, the

284 complexation of the PEO blocks of 904 is easier than that of 90R4 PEO blocks, located in the

285 inner part of the poloxamine arms. For steric reasons, the reverse poloxamine PEO blocks cannot

286 be fully complexed with CD molecules, so their mobility is not totally restricted and a glass

287 transition is detected.

288 Erosion and BSA release kinetics of 90R4/ $\mathbf{a}-\mathbf{C D}$ gels. According to the results shown above, 289 self-assembled hydrogels formed with reverse Tetronic 90R4 and $\alpha$-CD can be considered as

290 potential matrices for drug delivery systems. These gels showed an appropriate consistency, so

291 erosion kinetic studies were carried out in order to prove if the gels were suitable as release

292 matrices for sustained delivery purposes. On the other hand, the gels based on the direct

293 poloxamine (Tetronic 904) result too soft to be used for these purposes, so they were not tested. 
The release of $\alpha-\mathrm{CD}$ from the gels can be used as an indicator of the erosion kinetics of

295 the matrix, because $\alpha-\mathrm{CD}$ is one of the components of the gel structure. Figure $6 \mathrm{a}$ displays two

296 erosion kinetic curves at physiological $\mathrm{pH}$ and temperature for two different $90 \mathrm{R} 4 / \alpha-\mathrm{CD}$ gels.

297 One of them (TR15a10), showing a sustained slow erosion kinetics, is composed of 15\% of 90R4

298 and $10 \%(\mathrm{w} / \mathrm{w})$ of $\alpha-\mathrm{CD}$. The second one (TR25a10) was prepared using a higher amount of the

299 poloxamine, $25 \%$, and the same amount of $\alpha-\mathrm{CD}, 10 \%$. The latter is more viscous and thicker

300 than the former, as it was shown in Figure 4, although its erosion is considerably faster. TR15a10

301 gels contain more CD units per PEO block so the association between complexed PEO blocks is

302 more important than in TR25a10/CD gels. This gel is thicker because of the hydrophobic

303 interactions between its PPO blocks, which yield more viscous aggregates. The disaggregation of

304 polyrotaxane structures in reverse type poloxamers (Pluronics) and poloxamines (Tetronics) is

305 not as fast as the rupture of PPO-PPO interactions because the cyclodextrin molecules must slide

306 across the copolymer chain to break the complexes. Consequently, the erosion kinetics of

307 TR25a10 gels is significantly faster than that of TR15a10.

308 The disaggregation behaviours in different $\mathrm{pH}$ media were studied for the same gel

309 (Figure 6b). The erosion kinetics of three different samples of TR25a10 were evaluated in acidic,

310 neutral and basic solutions. The dissolution of the gels at $\mathrm{pH} 1$ and 7 is nearly the same, despite

311 the fact that Tetronic molecules are protonated at acidic $\mathrm{pH}$, so the interactions between Tetronic

312 molecules should change (Gonzalez-Lopez et al., 2008). Nevertheless, given the size of the

313 poloxamine molecules, it seems to be quite a small effect in this case. In contrast, the sample

314 shows a faster erosion process at $\mathrm{pH}$ 13. The main reason for this significantly behaviour may be

315 the ionization of the $\mathrm{CD}$ (Maeztu et al., 2011). At basic $\mathrm{pH}$, the external hydroxyl groups of the

316 CD molecules are deprotonated. For the PEO complexed blocks, the cyclodextrin rings can start

317 to repel each other, so the stability of the rotaxane clusters decreases. 
The erosion kinetics is almost the same at acidic and neutral $\mathrm{pH}$ : by tuning the hydrogel

319 compositions, the dismantling process can occur between two and five hours. A sustained drug

320 release is a promising feature of these systems which should be studied further. To conclude this

321 investigation, we decided to test the release of bovine serum albumin (BSA) as a model protein in

322 simulated physiological conditions. Figure 7 shows the release of BSA from TR25a10 and

323 TR15a10 gels as a function of time, at $\mathrm{pH} 7$ and $37^{\circ} \mathrm{C}$. The release process of BSA from T25a10

324 is faster than that of T15a10. This is consistent with the gel erosion profiles. As can be seen, both

325 profiles, i.e. those of the released molecule (BSA) (Figure 7) and the erosion ones (obtained by

326 measuring the $\alpha-\mathrm{CD}$ concentration in the aqueous release medium) (Figure 6a), are very similar.

327 This indicates that the release of substances from these gels is dominated by the erosion of the gel

328 matrix. As it was expected, a large molecule such as BSA (ca. $66.5 \mathrm{kDa}$ ) is mainly released as the

329 assembled $\mathrm{CD} /$ poloxamers structures are dismantled.

330

331 CONCLUSIONS

332 Both poloxamines, direct and reverse, can produce supramolecular gels when interacting with $\alpha$ -

$333 \mathrm{CD}$ in aqueous media. The comparison between the erosion profiles and the BSA release kinetics

334 for $90 \mathrm{R} 4 / \alpha-\mathrm{CD}$ gels proves that they can be applied for sustained release during short periods of

335 time. Although Tetronic 904 gels (i.e. the direct poloxamine) are not firm enough for this study,

336 they could be used for other types of release matrices applications such as ointments. Taking into

337 account that both PEO/PPO copolymers and $\alpha-\mathrm{CD}$ seem to be biocompatible, these gels can be

338 promising for biomedical applications.

339 Besides, it has to be remarked that the viscosity and the gel-to-sol transition temperatures

340 of these mixtures can be tailored by varying the poloxamine/ $\alpha$-CD ratios, so they can be tuned to

341 change at physiological temperatures (see Figures 2 and 3), in order to be applied for in situ 
342 gelling. An additional important consequence is that the erosion kinetics of the gel, i.e. its

343 disaggregation speed, can be also tuned by varying the copolymer/ $\alpha-\mathrm{CD}$ ratio.

\section{ACKNOWLEDGEMENTS}

346 The authors acknowledge the financial aid from the Ministerio de Ciencia e Innovación (project

347 MAT2007-65752) and Universidad de Navarra (PIUNA). E.L. thanks for a Gobierno de Navarra

348 grant (Plan de Formación y de I+D). We are also grateful to G. Tardajos (Universidad

349 Complutense) for her help with the NMR results and to C. Cesteros (Universidad del País Vasco

$350 \mathrm{UPV} / \mathrm{EHU}$ ) for DSC measurements.

351

352

\section{APPENDIX A: SUPPLEMENTARY DATA}

353 Figures A1-A7 and Table A1.

355 REFERENCES

356

357

358

359

360

361

362

363

364

365

366

367

368

369
Alexandridis P. \& Hatton T. (1995). Poly(ethylene oxide)-poly(propylene oxide)-poly(ethylene oxide) block copolymer surfactants in aqueous solutions and at interfaces: thermodynamics, structure, dynamics, and modeling. Colloids and Surfaces.A, Physicochemical and Engineering Aspects 96, 1-46.

Alvarez-Lorenzo C., Gonzalez-Lopez J., Fernandez-Tarrio M., Sandez-Macho I., \& Concheiro A. (2007). Tetronic micellization, gelation and drug solubilization: Influence of $\mathrm{pH}$ and ionic strength. European Journal of Pharmaceutics and Biopharmaceutics 66, 244-252.

Chung J., Kang T., \& Kwak S. (2007). Guest-free self-assembly of alpha-cyclodextrins leading to channel-type nanofibrils as mesoporous framework. Langmuir 23, 12366-12370.

Dodziuk, H. (2006; 2006). Cyclodextrins and Their Complexes: Chemistry, Analytical Methods,Applications.. : Wiley-VCH Verlag GmbH \& Co. KGaA.

Gonzalez-Lopez J., Alvarez-Lorenzo C., Taboada P., Sosnik A., Sandez-Macho I., \& Concheiro A. (2008). Self-associative behavior and drug-solubilizing ability of poloxamine (Tetronic) block copolymers. Langmuir 24, 10688-10697. 

Mahammed S., Sbille B., Gillet B., Beloeil J., Ringard C., Rosilio V., Poupaert J., \& Couvreur P. (2006). New self-assembled nanogels based on host-guest interactions: characterization and drug loading. Journal of Controlled Release 111, 316-324.

374 Harada A. (1998). Polyrotaxanes. Acta Polymerica 49, 3-17.

375 Harada A., Li J., \& Kamachi M. (1992). The molecular necklace - a rotaxane containing many 376 threaded alpha-cyclodextrin. Nature 356, 325-327.

377 Harada A., Okada M., Li J., \& Kamachi M. (1995). Preparation and characterization of inclusion

378 complexes of inclusion complexes of poly(propylene glycol) with cyclodextrins. Macromolecules $37928,8406-8411$.

380 Harada A., Takashima Y., \& Yamaguchi H. (2009). Cyclodextrin-based supramolecular 381 polymers. Chemical Society Reviews 38, 875-882.

382 Harada A. (1997). Construction of supramolecular structures from cyclodextrins, polymers.

383 Carbohydrate Polymers 34, 183-188.

384 Ko D., Shinde U., Yeon B., \& Jeong B. (2013). Recent progress of in situ formed gels for 385 biomedical applications. Progress in Polymer Science 38, 672-701.

386 Koopmans C. \& Ritter H. (2008). Formation of Physical Hydrogels via Host-Guest Interactions 387 of beta-Cyclodextrin Polymers and Copolymers Bearing Adamantyl Groups. Macromolecules 41, 388 7418-7422.

389 Larrañeta E. \& Isasi J. R. (2012). Self-assembled Supramolecular Gels of Reverse Poloxamers 390 and Cyclodextrins. Langmuir 28, 12457-12462.

391 Larrañeta E. \& Isasi J. R. (2013). Phase behavior of reverse poloxamers and poloxamines in 392 water. Langmuir 29, 1045-53.

393 Li J., Li X., Ni X., Wang X., Li H., \& Leong K. (2006). Self-assembled supramolecular 394 hydrogels formed by biodegradable PEO-PHB-PEO triblock copolymers and alpha-cyclodextrin 395 for controlled drug delivery. Biomaterials 27, 4132-4140.

Li J., Li X., Toh K., Ni X., Zhou Z., \& Leong K. (2001). Inclusion complexation and formation of polypseudorotaxanes between poly[(ethylene oxide)-ran-(propylene oxide)] and cyclodextrins. Macromolecules 34, 8829-8831. and applications for drug and gene delivery. Advanced Drug Delivery Reviews 60, 1000-1017.

401 Li J., Ni X., \& Leong K. (2003a). Injectable drug-delivery systems based on supramolecular 402 hydrogels formed by poly(ethylene oxide) and alpha-cyclodextrin. Journal of Biomedical 403 Materials Research 65A, 196-202. 

polypseudorotaxanes based on block-selected inclusion complexation between poly(propylene oxide)-poly(ethylene oxide)-poly(propylene oxide) triblock copolymers and alpha-cyclodextrin. Journal of the American Chemical Society 125, 1788-1795.

Lin Y., Li L., \& Li G. (2013). A new supramolecular gel via host-guest complexation with cucurbit[8] uril and N-(4-diethylaminobenzyl)chitosan. Carbohydrate Polymers 92, 429-34.

410 Maeztu R., Tardajos G., \& Gonzalez Gaitano G. (2011). Determination of the ionization constants of natural cyclodextrins by high-resolution (1)H-NMR and photon correlation spectroscopy. Journal of Inclusion Phenomena and Molecular Recognition in Chemistry 69, 361367.

Na G.C., Yuan B.O., Stevens, H.J.Jr., Weekley B.S., Rajagopalan N. (1999). Cloud point of nonionic surfactants: modulation with pharmaceutical excipients. Pharmaceutical Research 16, 562568.

Ni X., Cheng A., \& Li J. (2009). Supramolecular hydrogels based on self-assembly between Research.Part A 88A, 1031-1036.

Nielsen A., Steffensen K., \& Larsen K. (2009). Self-assembling microparticles with controllable disruption properties based on cyclodextrin interactions. Colloids and Surfaces.B, Biointerfaces 73, 267-275.

Perry C., Hebraud P., Gernigon V., Brochon C., \& Lapp A. (2011). Pluronic and betacyclodextrin in water: from swollen micelles to self-assembled crystalline platelets. Soft Matter 7, 3502-3512.

Plestil J., Pospisil H., Sikora A., Krakovsky I., \& Kuklin A. (2003). Small-angle neutron scattering and differential scanning calorimetry study of associative behaviour of branched poly(ethylene oxide)/poly(propylene oxide) copolymer in aqueous solution. Journal of Applied Crystallography 36, 970-975.

430 Simões S.M.N., Veiga F., Torres-Labandeira J.J., Ribeiro A.C.F., Concheiro A., Alvarez-Lorenzo C. (2013). Poloxamine-cyclodextrin-simvastatin supramolecular systems promote osteoblast differentiation of mesenchymal stem cells. Macromolecular Bioscience 13, 723-734.

Tan L., Liu Y., Ha W., Ding L., Peng S., Zhang S., \& Li B. (2012). Stimuli-induced gel-sol transition of multi-sensitive supramolecular $\beta$-cyclodextrin grafted alginate/ferrocene modified pluronic hydrogel. Soft Matter 8, 5746-5749.

Trong L., Djabourov M., \& Ponton A. (2008). Mechanisms of micellization and rheology of PEO-PPO-PEO triblock copolymers with various architectures. Journal of Colloid and Interface Science 328, 278-287. 
442 van de Manakker F., Vermonden T., van Nostrum C., \& Hennink W. (2008). Self-assembling 443 hydrogels based on beta-cyclodextrin/cholesterol inclusion complexes. Macromolecules 41, 444 1766-1773.

445 Van Tomme S., Storm G., \& Hennink W. (2008). In situ gelling hydrogels for pharmaceutical 446 and biomedical applications. International Journal of Pharmaceutics 355, 1-18.

447 Wintgens V., Daoud Mahammed S., Gref R., Bouteiller L., \& Amiel C. (2008). Aqueous 448 polysaccharide associations mediated by beta-cyclodextrin polymers. Biomacromolecules 9 , 449 1434-1442.

450 Zhou Z. \& Chu B. (1994). Phase Behavior and Association Properties of Poly(oxypropylene)451 Poly(oxyethylene)-Poly(oxypropylene) Triblock Copolymer in Aqueous Solution.

452 Macromolecules 27, 2025-2033. 
$453 \quad$ Figures and Tables

454

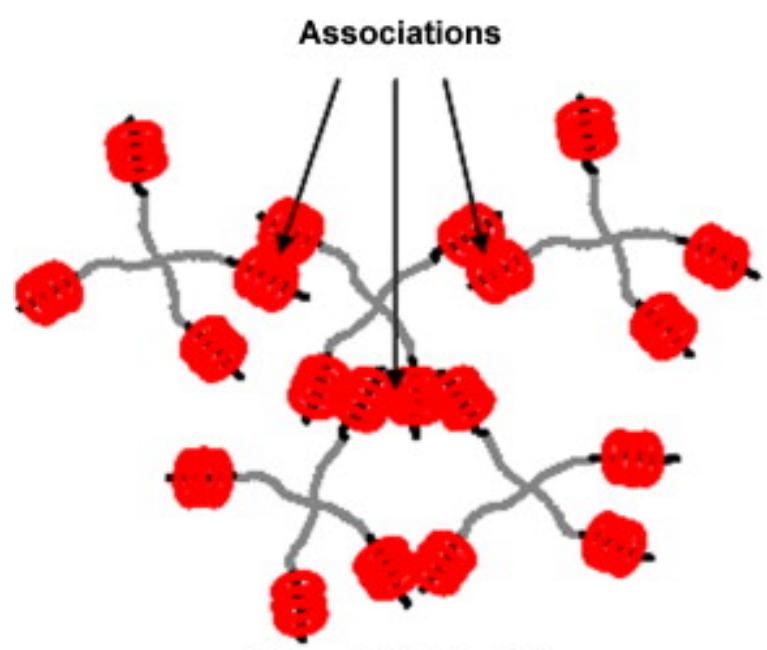

Tetronic $904+\alpha-C D$
Hydrophobic interactions

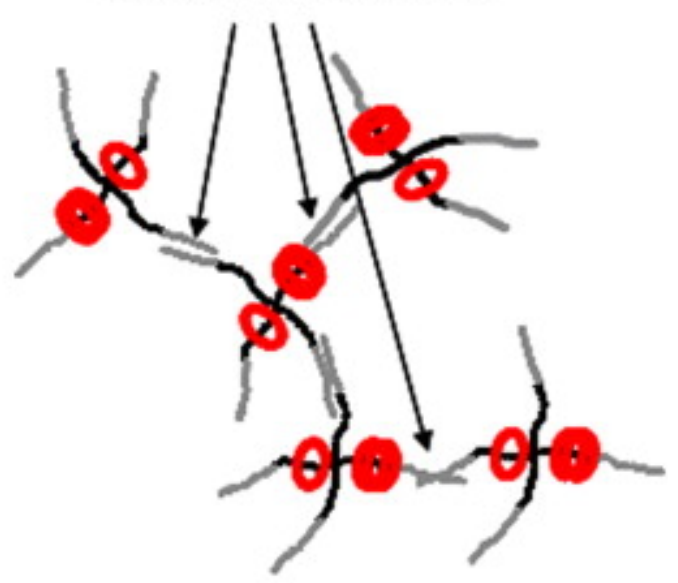

Tetronic 90R4 $+\alpha-C D$

456 Figure 1. Associations between $\alpha-C D(0)$ and normal 904 or reverse 90R4 Tetronics. 

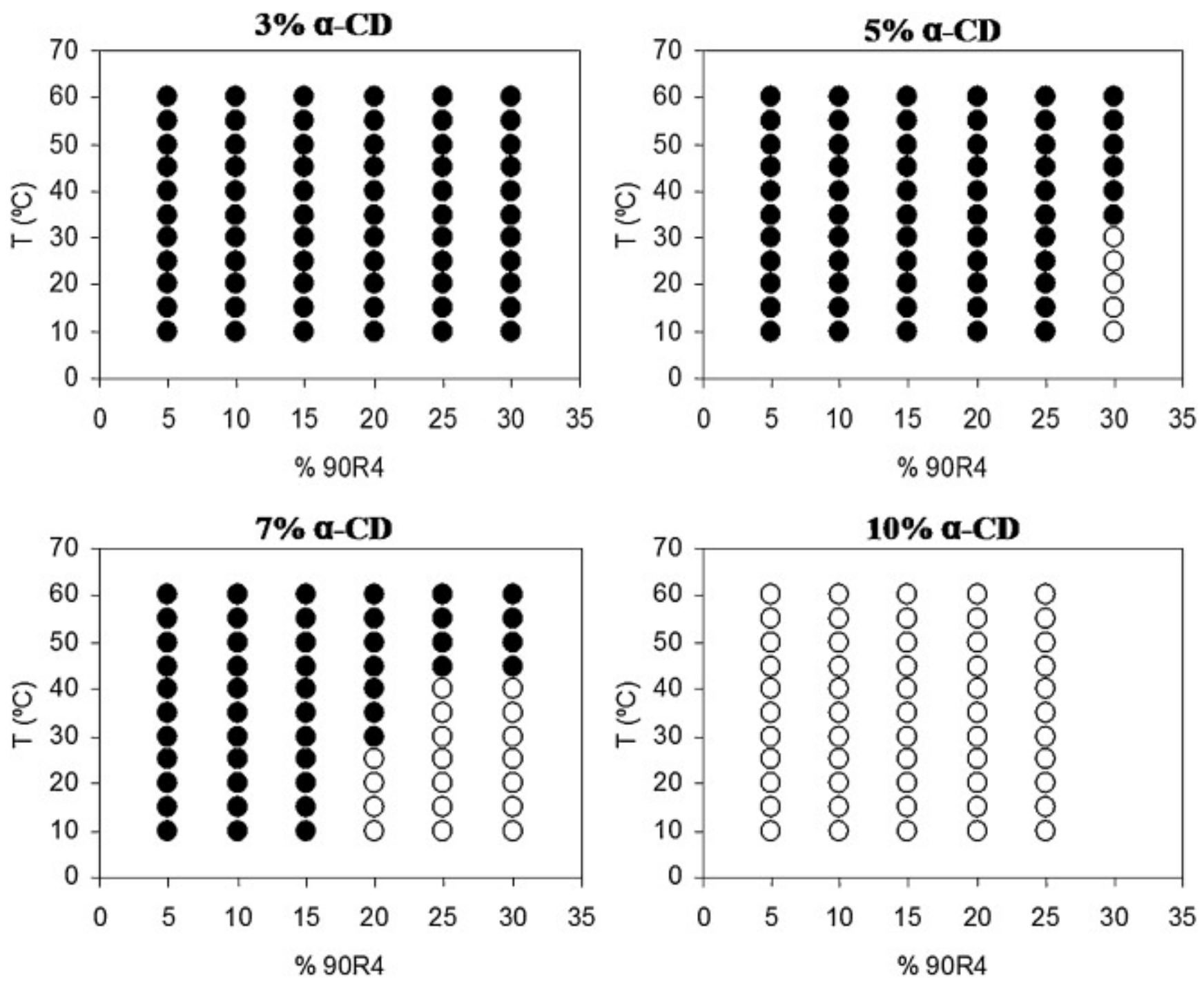

459 Figure 2. Temperature-concentration phase diagrams of aqueous reverse Tetronic 90R4 in the

460 presence of $\alpha$-CD. Sol phase $(\bullet)$ Gel phase (०) 

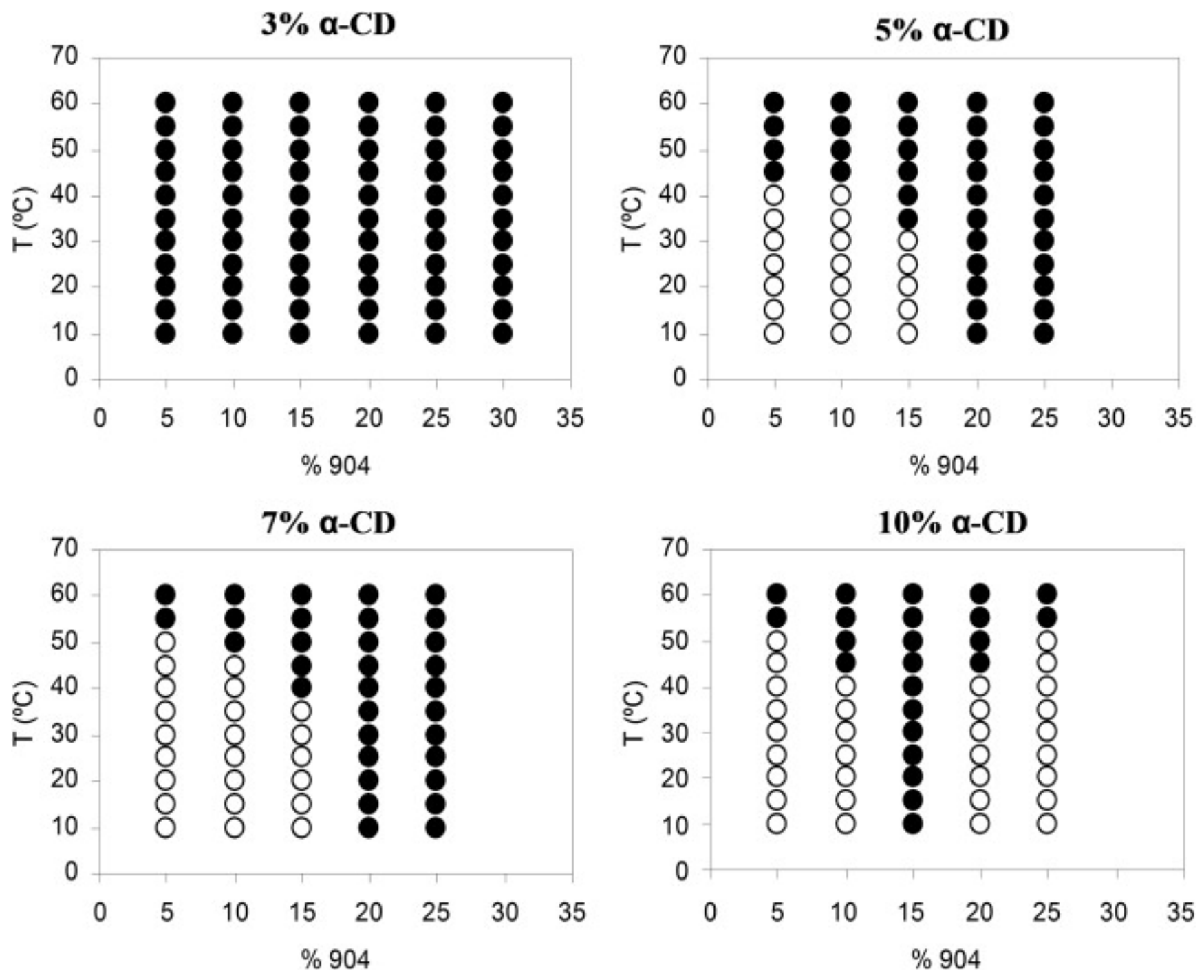

462

Figure 3. Temperature-concentration phase diagrams of aqueous normal Tetronic 904 in the 464 presence of $\alpha-C D$. Sol phase (•) Gel phase (०). 


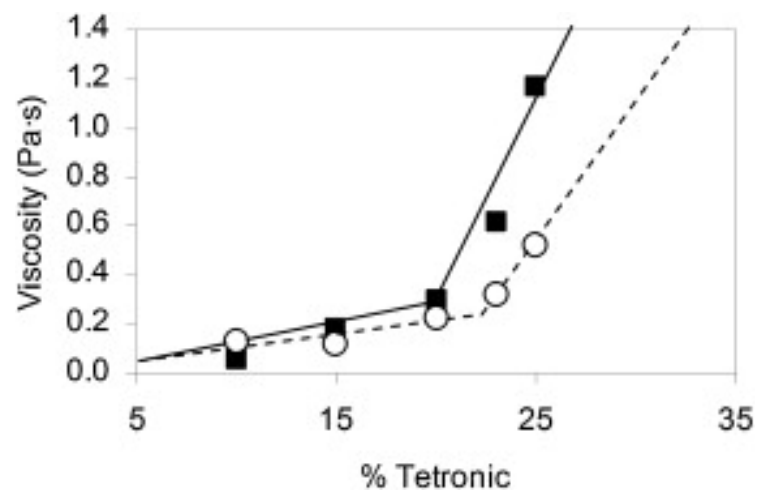

466 Figure 4. Viscosities of gels with $10 \%(\mathrm{w} / \mathrm{w})$ of $\alpha-\mathrm{CD}$ and different concentrations of Tetronic $467904(\circ)$ and Tetronic 90R4 ( $\mathbf{\square})$ measured at a constant shear rate of $40 \mathrm{~s}-1$.

468

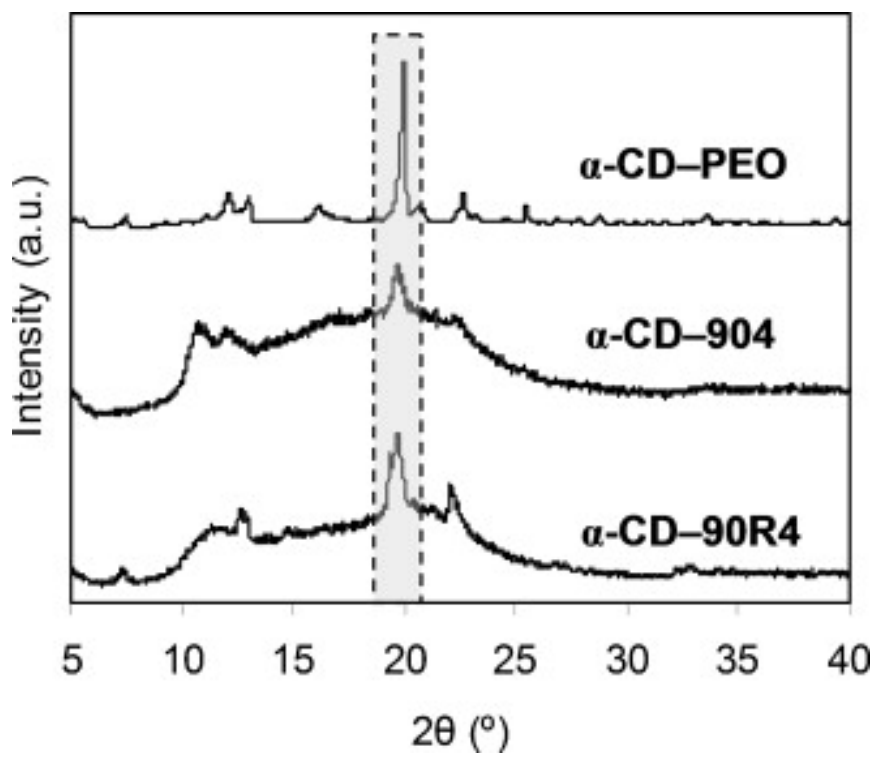

470 Figure 5. Wide-angle $\mathrm{X}$-ray diffractograms for $\alpha$-CD-PEO $(\mathrm{Mw}=400)$ complex, $\alpha$-CD-904 gel 471 containing $10 \%(\mathrm{w} / \mathrm{w})$ of CD and $25 \%$ of 904 , and $\alpha-C D-90 R 4$ gel containing $10 \%(w / w)$ of CD 472 and $25 \%$ of $90 \mathrm{R} 4$. 

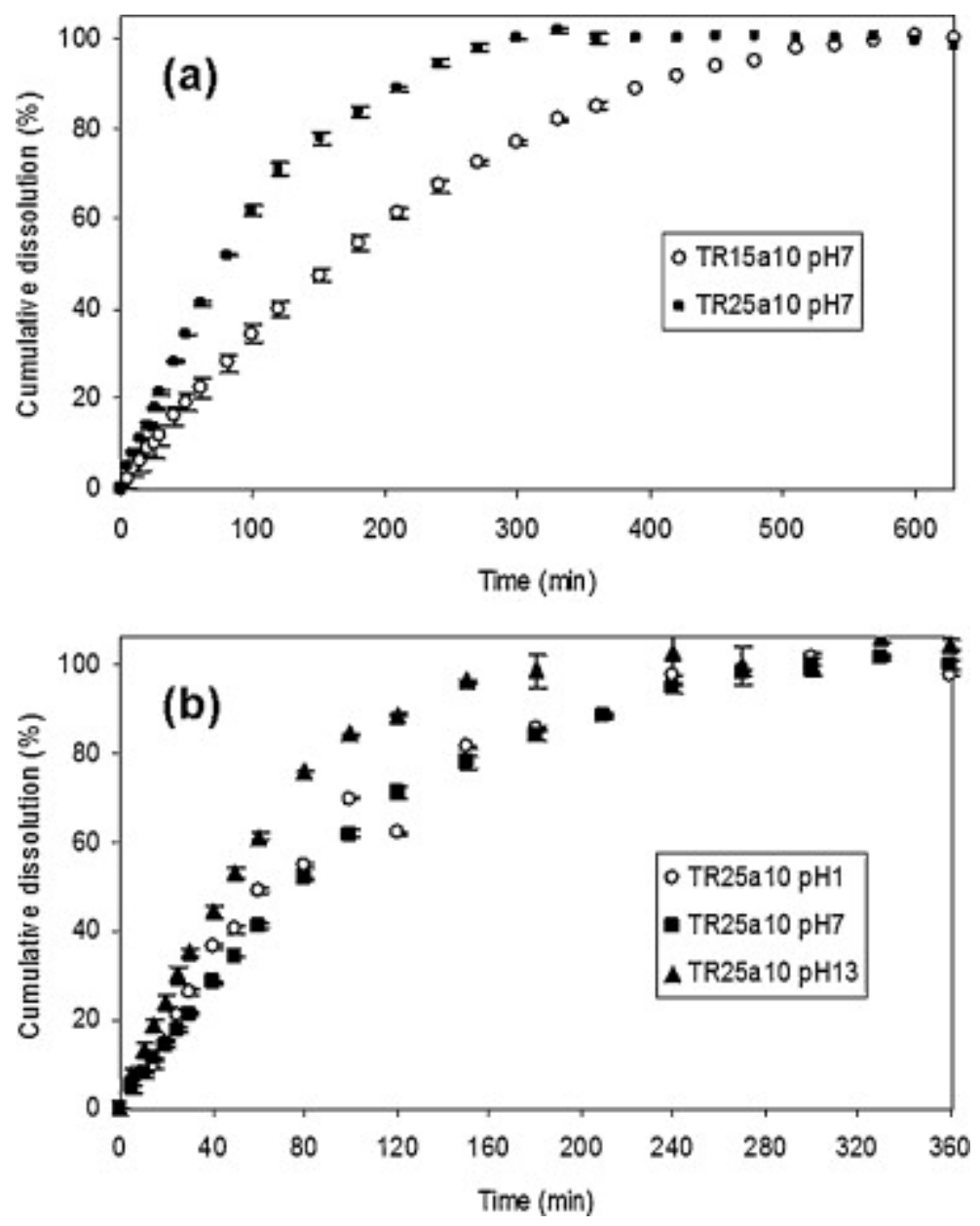

474 Figure 6. Erosion kinetics at $37{ }^{\circ} \mathrm{C}$ of two mixtures of $90 \mathrm{R} 4$ and $\alpha-\mathrm{CD}$, both containing $10 \%$ $475(\mathrm{w} / \mathrm{w})$ of $\alpha-\mathrm{CD}$ and $25 \%(\mathrm{w} / \mathrm{w})(\mathbf{a})$ or $15 \%(\mathrm{w} / \mathrm{w})(\mathrm{O})$ of $90 \mathrm{R} 4$ at $\mathrm{pH} 7(\mathrm{a})$, and three TR25a10 476 gels at different pHs: $1(\circ), 7(\mathbf{a})$ and $13(\mathrm{l})(\mathrm{b})$ (error bars for two replicates). 


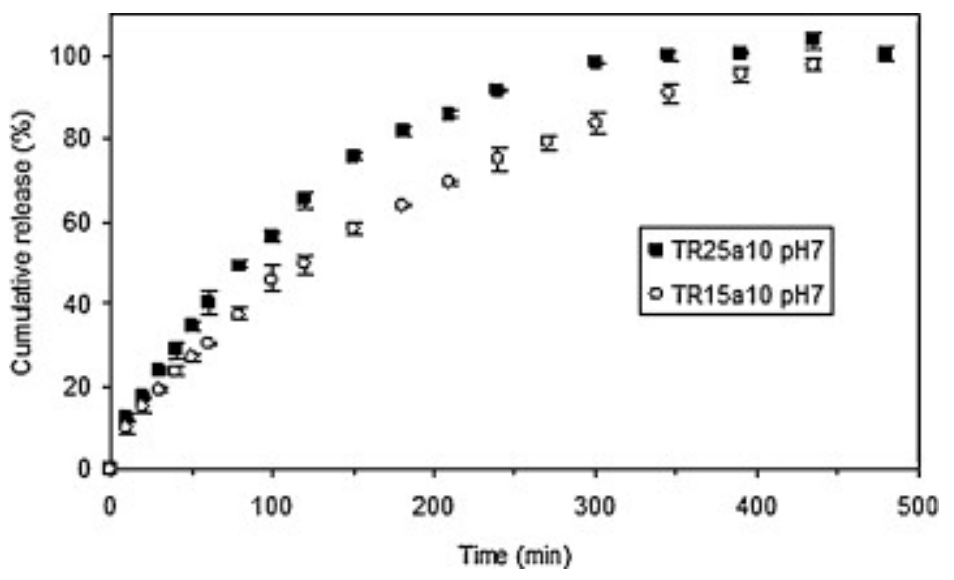

478 Figure 7. BSA release from two gels, both containing $10 \%(w / w)$ of $\alpha-C D$ and $25 \%(w / w)(\boldsymbol{m})$ or $47915 \%(\mathrm{w} / \mathrm{w})(\mathrm{O})$ of $90 \mathrm{R} 4$ at physiological conditions (error bars for two replicates).

480

481

482

Table 1.Tg for different complexes of 904 or 90R4 with $\alpha-C D$.

\begin{tabular}{|c|c|c|c|c|c|}
\hline \multirow[b]{2}{*}{ Sample name } & \multicolumn{3}{|c|}{ Mixture composition (\%) } & \multirow[b]{2}{*}{$\mathrm{EO} / \mathrm{CD}$} & \multirow[b]{2}{*}{$\operatorname{Tg}\left({ }^{\circ} \mathrm{C}\right)$} \\
\hline & Copolymer & $\alpha-C D$ & $\mathrm{H}_{2} \mathrm{O}$ & & \\
\hline$\alpha-C D$ & - & - & - & - & - \\
\hline T1a10 & 1.00 & 10.00 & 89.00 & 0.94 & - \\
\hline $\mathrm{T} 2 \mathrm{a} 10$ & 2.00 & 10.00 & 88.00 & 1.90 & - \\
\hline T3a10 & 3.00 & 10.00 & 87.00 & 2.73 & - \\
\hline T4a10 & 4.00 & 10.00 & 86.00 & 3.58 & -56.5 \\
\hline T10a10 & 10.00 & 10.00 & 80.00 & 9.04 & -55.4 \\
\hline $\mathrm{T} 20 \mathrm{a} 10$ & 20.00 & 10.00 & 70.00 & 18.08 & -59.0 \\
\hline 904 & 100 & 0 & 0 & - & -58.3 \\
\hline TR1a10 & 1.00 & 10.00 & 89.00 & 0.94 & -66.1 \\
\hline TR2a10 & 2.00 & 10.00 & 88.00 & 1.90 & -60.1 \\
\hline TR3a10 & 3.00 & 10.00 & 87.00 & 2.73 & -61.3 \\
\hline TR4a10 & 4.00 & 10.00 & 86.00 & 3.58 & -59.8 \\
\hline TR10a10 & 10.00 & 10.00 & 80.00 & 9.04 & -64.7 \\
\hline TR20a10 & 20.00 & 10.00 & 70.00 & 18.08 & -65.6 \\
\hline $90 \mathrm{R} 4$ & 100 & 0 & 0 & - & -62.8 \\
\hline
\end{tabular}


Title: Non-covalent hydrogels of cyclodextrins and poloxamines for the controlled release of proteins

489

490

491

492

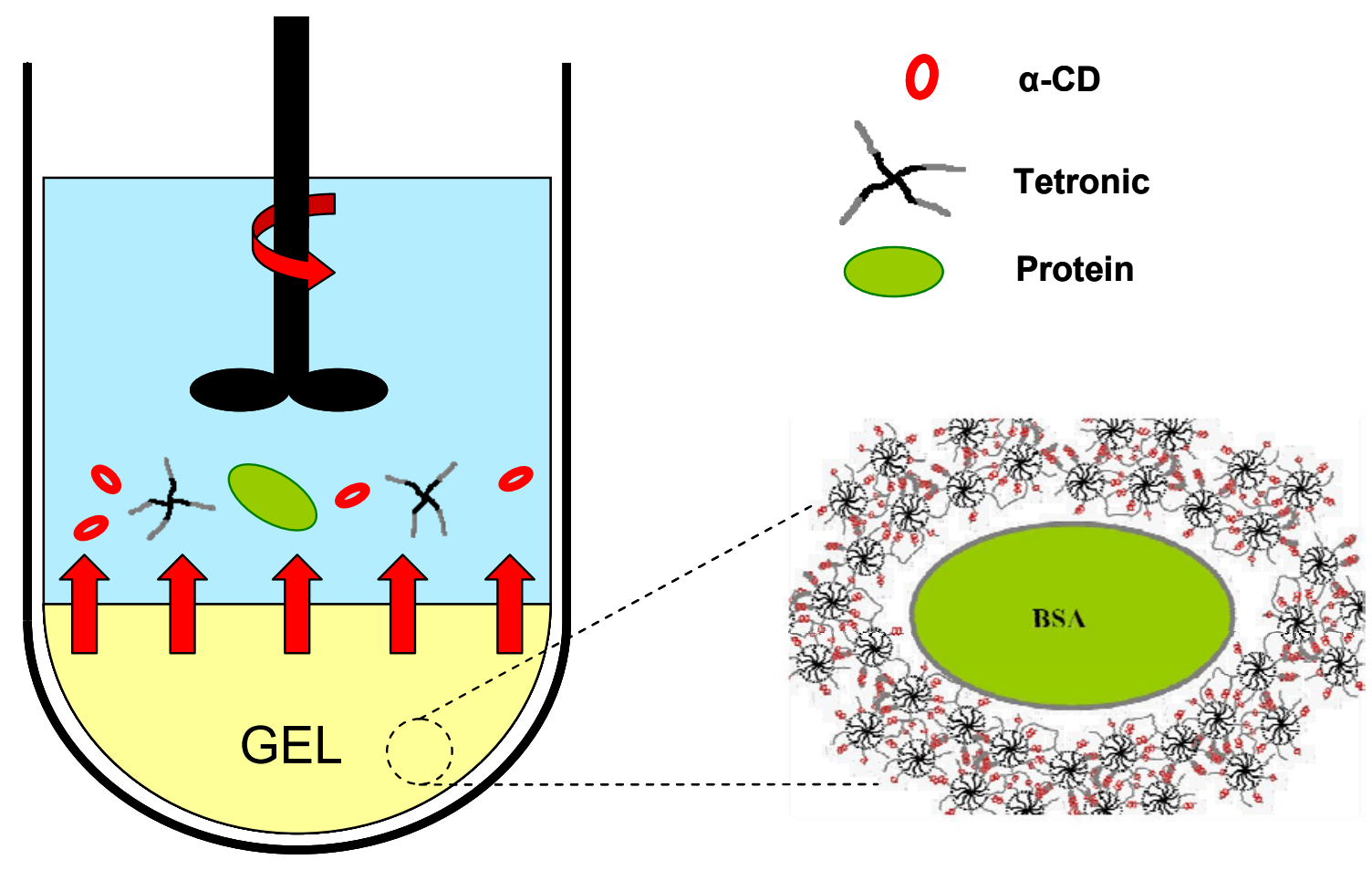

493

494

495

496

497

498 
500

501 Title: Non-covalent hydrogels of cyclodextrins and poloxamines for the controlled release of 502 proteins

503 Authors: E. Larrañeta, J.R. Isasi

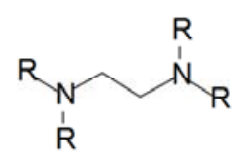

(a) $\mathrm{R}=\mathrm{A}$ of $\mathrm{O}_{\mathrm{x}} \mathrm{H}$

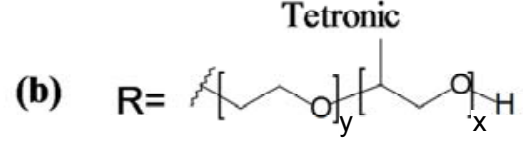

Tetronic R

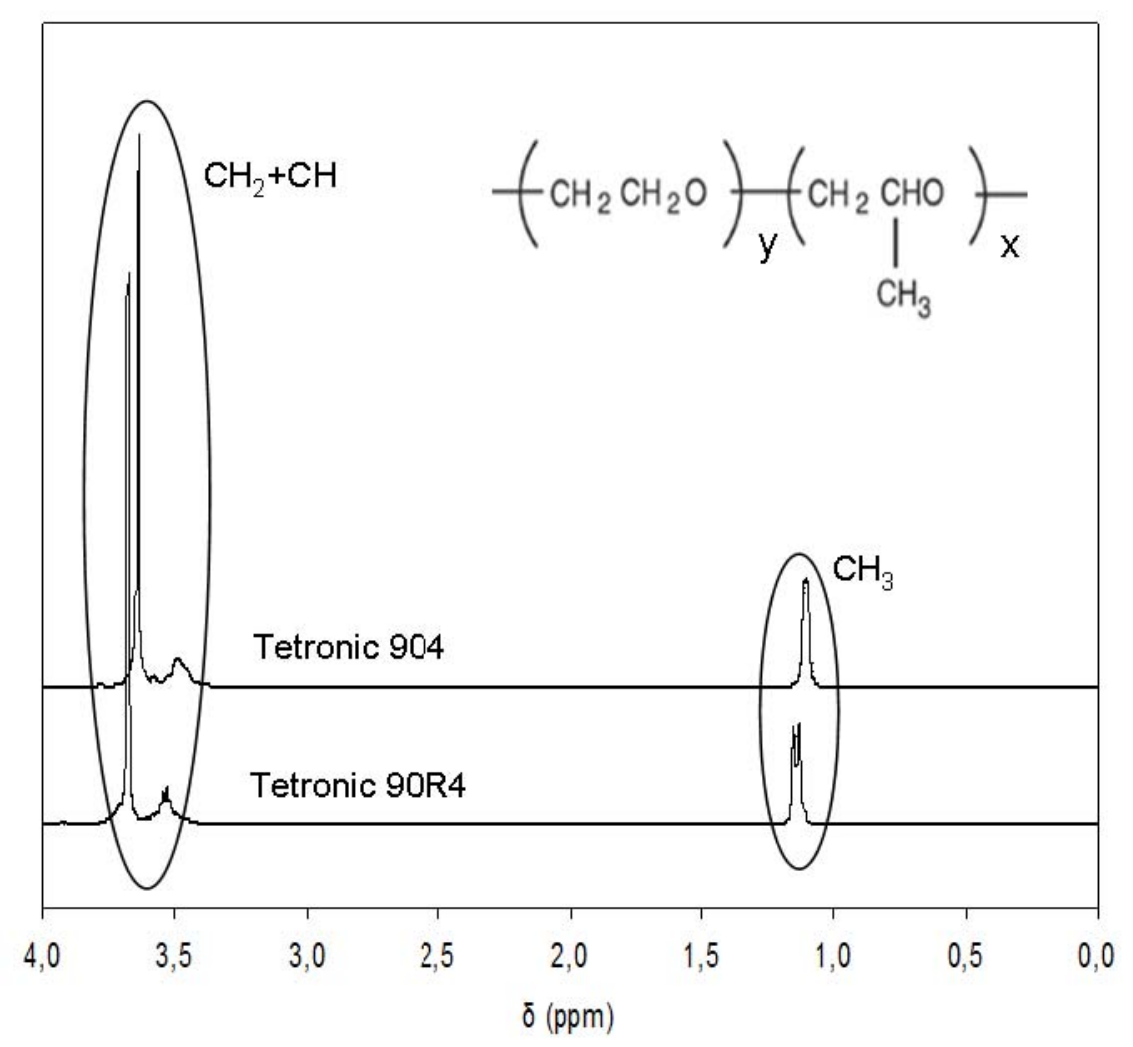


Figure A1. ${ }^{1} \mathrm{H}-\mathrm{NMR}$ spectra of Tetronic 904 and Tetronic 90R4. For each copolymer, $x$ and $y$ values are calculated using the molecular weight given by the manufacturer, the molecular weights of both units, and the ratio of both NMR signals.

\section{Determination of released amounts of BSA}

512 To determine the amount of BSA released three calibration curves are needed:

Fluorescence:

$$
\begin{aligned}
& \mathrm{I}_{\mathrm{f}}=\mathrm{K}_{\mathrm{f}}{ }^{(B S A)} \cdot[\mathrm{BSA}] \\
& \mathrm{A}^{(B S A)}=\mathrm{K}_{\mathrm{UV}}{ }^{(B S A)} \cdot[\mathrm{BSA}]
\end{aligned}
$$

$$
\text { UV-vis: }
$$$$
\mathrm{A}^{(90 R 4)}=\mathrm{K}_{\mathrm{UV}}{ }^{(90 R 4)} \cdot[90 \mathrm{R} 4]
$$

The samples collected by the dissolution testing device were evaluated both by $\mathbf{U V}$-vis at 280 $\mathbf{n m}$ and by fluorescence emission at $341 \mathrm{~nm}$ (excitation at $280 \mathrm{~nm}$ ). With these data, the following equations are obtained:

UV-vis:

$$
\mathrm{A}_{\text {sample }}=\mathrm{K}_{\mathrm{UV}}{ }^{(B S A)} \cdot[\mathrm{BSA}]+\mathrm{K}_{\mathrm{UV}}{ }^{(90 R 4)} \cdot[\text { [90R4] }
$$

Fluorescence:

$$
\mathrm{I}_{\mathrm{f}} / \mathrm{I}_{\mathrm{Q}}=\mathrm{K}_{\mathrm{Q}} \cdot[90 \mathrm{R} 4] \quad \rightarrow
$$

$$
\mathrm{I}_{\mathrm{Q}}=\mathrm{I}_{\mathrm{f}} /\left(\mathrm{K}_{\mathrm{Q}} \cdot[90 \mathrm{R} 4]\right)
$$

where $\mathbf{K}_{\mathbf{Q}}$ is the quenching Stern-Volmer constant at room temperature and $\mathbf{I}_{\mathbf{Q}}$ is the actual intensity measured for each sample and $\mathbf{I}_{\mathbf{f}}$ would be that in the absence of the quencher, i.e. the one we have in the calibration curve. case of the fluorescence signal expression, substituting $I_{f}$ from its calibration, we obtain two equations with two unknowns ([BSA] and [90R4]):

\section{Fluorescence:}

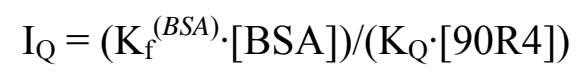




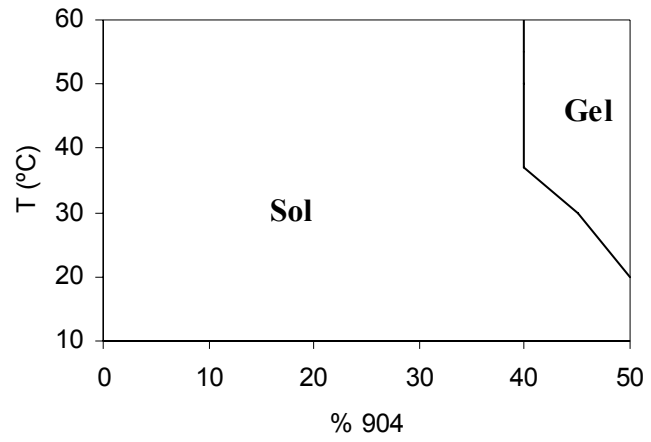

535 Figure A2. Sol-gel phase diagram for 904/water solutions as a function of the temperature.
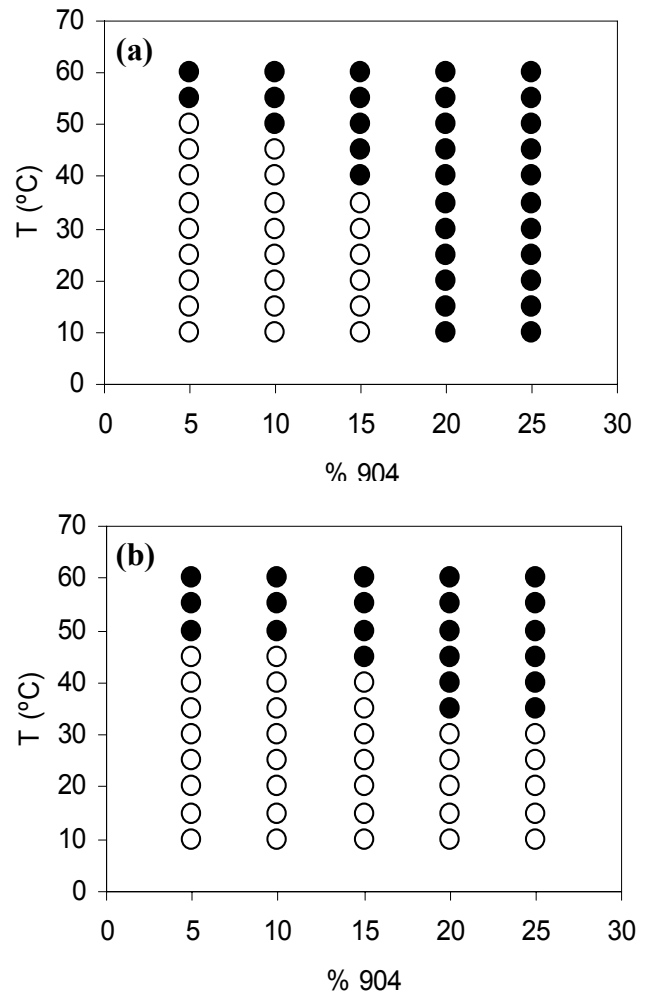

538 Figure A3. Variation of the temperature-concentration phase diagrams of aqueous Tetronic 904 in the 539 presence of $7 \%$ of $\alpha-C D$. Sol phase (•), gel phase (o). (a) First scan and (b) second scan.

541 Turbidity measurements. Turbidities of two mixtures containing $0.5 \%(\mathrm{w} / \mathrm{w})$ of the copolymer (Tetronic

542904 or Tetronic 90R4) and $1.5 \%(\mathrm{w} / \mathrm{w})$ of $\beta-\mathrm{CD}$ were measured using a JASCO V-630 UV-vis

543 spectrophotometer. All the measurements were carried out at a fixed wavelength of $600 \mathrm{~nm}$ as a function 544 of time. 


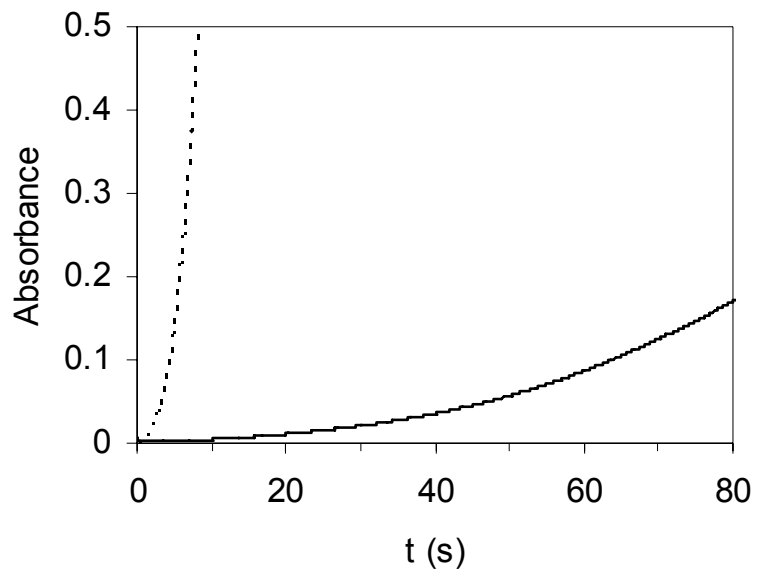

546 Figure A4. Turbidity of two mixtures containing $0.5 \%$ of copolymer, Tetronic 904 (solid line) and

547 Tetronic 90R4 (dashed line), and $1.5 \%$ of $\beta-\mathrm{CD}$.

\section{Characterization of complexes between $\beta-C D$ and 90R4 or 904}

550 Elemental analysis measurements. The elemental analysis were carried out with a Leco CHN 900 using

551 about $1 \mathrm{mg}$ of sample.

552 NMR measurements. The solid-state ${ }^{13} \mathrm{C}$ CP/MAS NMR spectra were measured using a Bruker AV-400

553 NMR Wide Bore with a sample spinning rate of $12 \mathrm{kHz}$ at room temperature. Cross-polarization spectra

554 were acquired with a $4 \mu$ s proton $90^{\circ}$ pulse, a $2.5 \mathrm{~ms}$ contact time, and a $5 \mathrm{~s}$ repetition time.

555

556 Table A1. Elemental analysis and NMR calculated PO/CD mole ratios for complexes of 90R4(TR) and

557 904(T) with $\beta-C D$

\begin{tabular}{ccccccccc}
\hline & & \multicolumn{3}{c}{$\begin{array}{c}\text { mixture } \\
\text { composition (\%) }\end{array}$} & \multicolumn{2}{c}{ elemental composition (\%) } & \\
\cline { 3 - 7 } sample name & copolymer & copolymer & $\beta-\mathrm{CD}$ & $\mathrm{H}_{2} \mathrm{O}$ & $\mathrm{C}$ & $\mathrm{H}$ & $\mathrm{O}$ & NMR \\
& & & & & & & & PO/CD ratio \\
\hline TR0.5b1.5 & $90 \mathrm{R} 4$ & 0.50 & 1.50 & 98.00 & 47.65 & 7.14 & 45.21 & 2.47 \\
TR1b1 & $90 \mathrm{R} 4$ & 1.00 & 1.00 & 98.00 & 47.90 & 7.14 & 44.97 & \\
T0.5b1.5 & 904 & 0.50 & 1.50 & 98.00 & 45.81 & 7.04 & 47.15 & 2.12 \\
T1b1 & 904 & 1.00 & 1.00 & 98.00 & 47.12 & 7.28 & 45.60 & \\
\hline
\end{tabular}




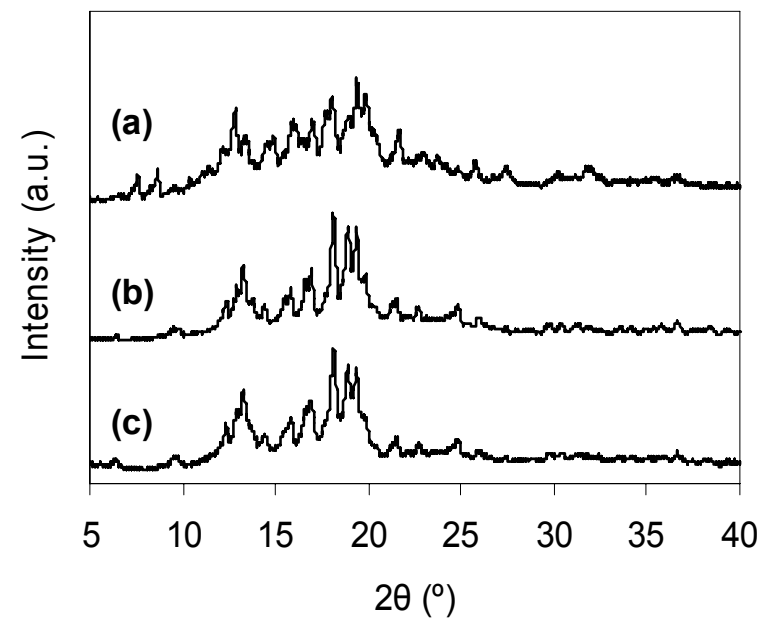

Figure A5. X-ray diffraction patterns of: $\beta$-CD (a), TR0.5b1.5 (b) and T0.5b1.5 (c).

\section{Rheological behavior of $\alpha-C D / 90 R 4$ and $\alpha-C D / 904$ gels}

568

569

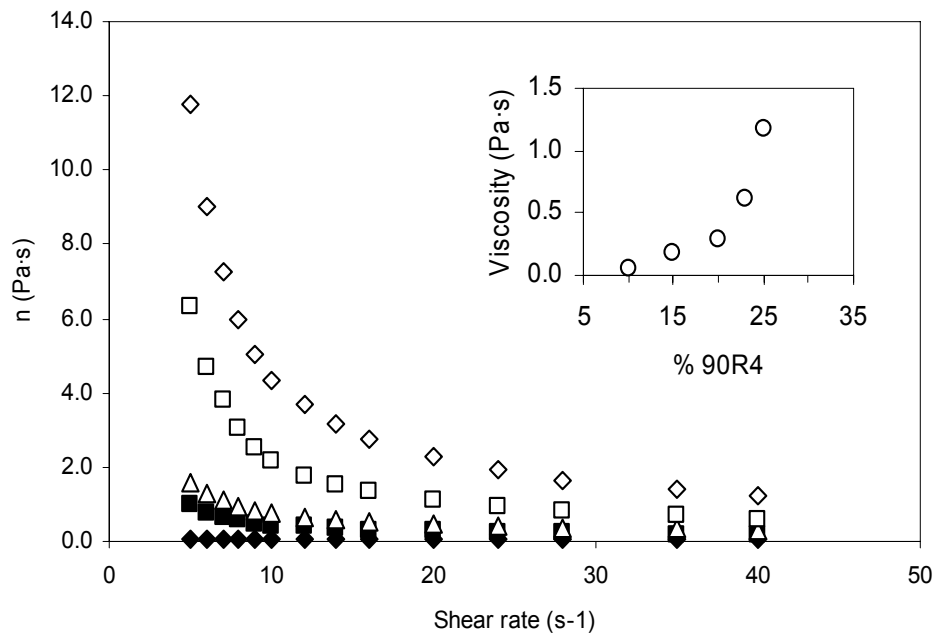

571 Figure A6. Viscosities of gels with 10\% (w/w) of $\alpha$-CD and different concentrations of 90R4: 10\% (४), $57215 \%(\square), 20 \%(\Delta), 23 \%(\square)$ and $25 \%(\diamond)$ as a function of shear rate. The inset shows the dependence of 573 the viscosity with the concentration of 90R4 measured at a constant shear rate of $40 \mathrm{~s}^{-1}$. 


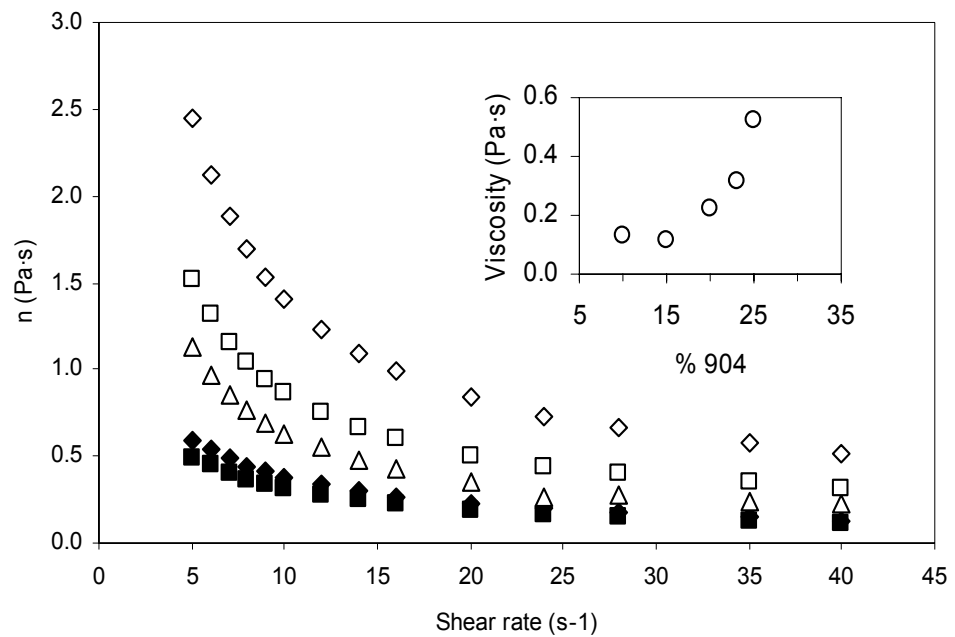

575

576 Figure A7. Viscosities of gels with 10\% (w/w) of $\alpha-C D$ and different concentrations of $904: 10 \%(\diamond)$, $57715 \%(\square), 20 \%(\Delta), 23 \%(\square)$ and $25 \%(\diamond)$ as a function of shear rate. The inset shows the dependence of 578 the viscosity with the concentration of $90 \mathrm{R} 4$ at a constant shear rate of $40 \mathrm{~s}^{-1}$.

579

580 